\title{
Rational design of a split flavin-based fluorescent reporter
}

Anna Yudenko, Anastasia Smolentseva, Ivan Maslov, Oleg Semenov, Ivan M. Goncharov, Vera V. Nazarenko, Nina L. Maliar, Valentin Borshchevskiy, Valentin Gordeliy, Alina Remeeva, Ivan Gushchin*

*E-mail for correspondence: ivan.gushchin@phystech.edu

\section{Supporting Information}

Tables S1-S2

Figures S1-S3 
Table S1. Amino acid sequences of the proteins studied in this work. Amino acids corresponding to the $\mathrm{CagFbFP}$ core are highlighted in yellow; TEV protease cleavage site is in light blue; SUMO is in dark blue; NZ and CZ leucine zippers are in violet and pink, respectively; mitochondrial targeting sequence is in light blue; calmodulin is in dark green and calmodulin-binding peptide is in brown.

\begin{tabular}{|c|c|}
\hline $\begin{array}{l}\text { Name of } \\
\text { the construct }\end{array}$ & Sequence \\
\hline CagFbFP & $\begin{array}{l}\text { MASGMIVTDAGADQPIVFVNRAFSTITGYAPNEVLGRNARFLQGPQTDAATVARLREAIAAARPIQ } \\
\text { ERILNYRKDGQPFWNQLSIS PVRDETGNVVAFVGVQTDVTAHHHHHH }\end{array}$ \\
\hline LOV58ins2 & $\begin{array}{l}\text { MASGMIVTDAGAGGDQP IVFVNRAFSTITGYAPNEVLGRNARFLQGPQTDAATVARLREAIAAARP } \\
\text { IQERI LNYRKDGQP FWNQLSISPVRDETGNVVAFVGVQTDVTAHHHHHH }\end{array}$ \\
\hline LOV58ins5 & $\begin{array}{l}\text { MASGMIVTDAGAGSGSGDQPIVFVNRAFSTITGYAPNEVLGRNARFLQGPQTDAATVARLREAIAA } \\
\text { ARPIQERI LNYRKDGQPFWNQLSISPVRDETGNVVAFVGVQTDVTAHHHHHH }\end{array}$ \\
\hline LOV58ins9 & $\begin{array}{l}\text { MASGMIVTDAGAGGSGGGSGGDQP IVFVNRAFST ITGYAPNEVLGRNARFLQGPQTDAATVARLRE } \\
\text { AIAAARPIQERI LNYRKDGQPFWNQLSISPVRDETGNVVAFVGVQTDVTAHHHHHH }\end{array}$ \\
\hline LOV91ins2 & $\begin{array}{l}\text { MASGMIVTDAGADQPIVFVNRAFSTITGYAPNEVLGRNARFLQGAGGQTDAATVARLREAIAAARP } \\
\text { IQERI LNYRKDGQP FWNQLSISPVRDETGNVVAFVGVQTDVTAHHHHHH }\end{array}$ \\
\hline LOV91ins5 & $\begin{array}{l}\text { MASGMIVTDAGADQPIVFVNRAFSTITGYAPNEVLGRNARFLQGAGSGSGQTDAATVARLREAIAA } \\
\text { ARPIQERI LNYRKDGQPFWNQLS ISPVRDETGNVVAFVGVQTDVTAHHHHHH }\end{array}$ \\
\hline LOV91ins9 & $\begin{array}{l}\text { MASGMIVTDAGADQPIVFVNRAFSTITGYAPNEVLGRNARFLQGAGGSGGGSGGQTDAATVARLRE } \\
\text { AIAAARPIQERI LNYRKDGQPFWNQLSIS PVRDETGNVVAFVGVQTDVTAHHHHHH }\end{array}$ \\
\hline LOV137ins2 & $\begin{array}{l}\text { MASGMIVTDAGADQPIVFVNRAFSTITGYAPNEVLGRNARFLQGPQTDAATVARLREAIAAARPIQ } \\
\text { ERILNYRKDGQPFWNQLSIS PVRDEGGTGNVVAFVGVQTDVTAHHHHHH }\end{array}$ \\
\hline LOV137ins5 & $\begin{array}{l}\text { MASGMIVTDAGADQPIVFVNRAFSTITGYAPNEVLGRNARFLQGPQTDAATVARLREAIAAARPIQ } \\
\text { ERILNYRKDGQPFWNQLS ISPVRDEGSGSGTGNVVAFVGVQTDVTAHHHHHH }\end{array}$ \\
\hline LOV137ins9 & $\begin{array}{l}\text { MASGMIVTDAGADQPIVFVNRAFSTITGYAPNEVLGRNARFLQGPQTDAATVARLREAIAAARPIQ } \\
\text { ERILNYRKDGQPFWNQLSISPVRDEGGSGGGSGGTGNVVAFVGVQTDVTAHHHHHH }\end{array}$ \\
\hline LOV58insTEV & $\begin{array}{l}\text { MASGMIVTDAGAGGGSGGSGSENLYFQSGGSGGGGSGDQP IVFVNRAFST ITGYAPNEVLGRNARF } \\
\text { LQGPQTDAATVARLREAIAAARPIQERI LNYRKDGQPFWNQLS ISPVRDETGNVVAFVGVQTDVTA } \\
\text { HHHHHH }\end{array}$ \\
\hline LOV91insTEV & $\begin{array}{l}\text { MASGMIVTDAGADQPIVFVNRAFSTITGYAPNEVLGRNARFLQGAGGGSGGSGSENLYFQSGGSGG } \\
\text { SGSGQTDAATVARLREA IAAARPIQERI LNYRKDGQPFWNQLS ISPVRDETGNVVAFVGVQTDVTA } \\
\text { HнHнHн }\end{array}$ \\
\hline LOV137insTEV & $\begin{array}{l}\text { MASGMIVTDAGADQPIVFVNRAFSTITGYAPNEVLGRNARFLQGPQTDAATVARLREAIAAARPIQ } \\
\text { ERILNYRKDGQPFWNQLSIS PVRDEGGGSGGSGSENLYFQSGGSGGGGSGTGNVVAFVGVQTDVTA } \\
\text { HHHHHH }\end{array}$ \\
\hline $\begin{array}{l}\text { SUMO-LOV48- } \\
58-\mathrm{NZ}\end{array}$ & $\begin{array}{l}\text { MHHHHHHMGSLQDSEVNQEAKPEVKPEVKPETHINLKVSDGSSEIFFKIKKTTPLRRLMEAFAKRQ } \\
\text { GKEMDSLTFLYDGIEIQADQTPEDLDMEDNDI IEAHREQIGGENLYFQGASGMIVTDAGAGGSGGA } \\
\text { LKKELQANKKELAQLKWELQALKKELAQ }\end{array}$ \\
\hline $\begin{array}{l}\text { SUMO-LOV48- } \\
58\end{array}$ & $\begin{array}{l}\text { MHHHHHHMGSLQDSEVNQEAKPEVKPEVKPETHINLKVSDGSSEIFFKIKKTTPLRRLMEAFAKRQ } \\
\text { GKEMDSLTFLYDGIEIQADQTPEDLDMEDNDI IEAHREQIGGENLYFQGASGMIVTDAGA }\end{array}$ \\
\hline $\begin{array}{l}\text { CZ-LOV59-153- } \\
\text { His }\end{array}$ & $\begin{array}{l}\text { MEQLEKKLQALEKKLAQLEWKNQALEKKLAQGGSGGDQPIVFVNRAFSTITGYAPNEVLGRNARFL } \\
\text { QGPQTDAATVARLREAIAAARPIQERILNYRKDGQP FWNQLS IS PVRDETGNVVAFVGVQTDVTAH } \\
\text { HHHHH }\end{array}$ \\
\hline $\begin{array}{l}\text { SUMO-LOV48- } \\
91-\mathrm{NZ}\end{array}$ & $\begin{array}{l}\text { MHHHHHHMGSLQDSEVNQEAKPEVKPEVKPETHINLKVSDGSSEIFFKIKKTTPLRRLMEAFAKRQ } \\
\text { GKEMDSLTFLYDGIEIQADQTPEDLDMEDNDI IEAHREQIGGENLYFQGASGMIVTDAGADQPIVF } \\
\text { VNRAFSTITGYAPNEVLGRNARFLQGAGGSGGALKKELQANKKELAQLKWELQALKKELAQ }\end{array}$ \\
\hline
\end{tabular}




\begin{tabular}{|c|c|}
\hline $\begin{array}{l}\text { SUMO-LOV48- } \\
91\end{array}$ & $\begin{array}{l}\text { MHHHHHHMGSLQDSEVNQEAKPEVKPEVKPETHINLKVSDGSSEIFFKIKKTTPLRRLMEAFAKRQ } \\
\text { GKEMDSLTFLYDGIEIQADQTPEDLDMEDNDI IEAHREQIGGENLYFQGASGMIVTDAGADQPIVF } \\
\text { VNRAFSTITGYAPNEVLGRNARFLQGA }\end{array}$ \\
\hline $\begin{array}{l}\text { CZ-LOV92-153- } \\
\text { His }\end{array}$ & $\begin{array}{l}\text { MEQLEKKLQALEKKLAQLEWKNQALEKKLAQGGSGGQTDAATVARLREAIAAARP IQERI LNYRKD } \\
\text { GQPFWNQLSISPVRDETGNVVAFVGVQTDVTAHHHHHH }\end{array}$ \\
\hline $\begin{array}{l}\text { SUMO-LOV48- } \\
137-\mathrm{NZ}\end{array}$ & $\begin{array}{l}\text { MHHHHHHMGSLQDSEVNQEAKPEVKPEVKPETHINLKVSDGSSEIFFK IKKTTPLRRLMEAFAKRQ } \\
\text { GKEMDSLTFLYDGIEIQADQTPEDLDMEDNDI IEAHREQIGGENLYFQGASGMIVTDAGADQP IVF } \\
\text { VNRAFST ITGYAPNEVLGRNARFLQGPQTDAATVARLREAIAAARP IQERI LNYRKDGQPFWNQLS } \\
\text { ISPVRDEGGSGGALKKELQANKKELAQLKWELQALKKELAQ }\end{array}$ \\
\hline $\begin{array}{l}\text { SUMO-LOV48- } \\
137\end{array}$ & $\begin{array}{l}\text { MHHHHHHMGSLQDSEVNQEAKPEVKPEVKPETHINLKVSDGSSEIFFK IKKTTPLRRLMEAFAKRQ } \\
\text { GKEMDSLTFLYDGIEIQADQTPEDLDMEDNDI IEAHREQIGGENLYFQGASGMIVTDAGADQPIVF } \\
\text { VNRAFSTITGYAPNEVLGRNARFLQGPQTDAATVARLREAIAAARP IQERI LNYRKDGQPFWNQLS } \\
\text { ISPVRDE }\end{array}$ \\
\hline $\begin{array}{l}\text { CZ-LOV138- } \\
153 \text {-His }\end{array}$ & MEQLEKKLQALEKKLAQLEWKNQALEKKLAQGGSGGTGNVVAFVGVQTDVTAHHHHHH \\
\hline MTS-CagFbFP & $\begin{array}{l}\text { MSVLTPLLLRGLTGSARRLPVPRAKI HSLGPPVMASGMIVTDAGADQP IVFVNRAFSTITGYAPNE } \\
\text { VLGRNARFLQGPQTDAATVARLREAIAAARPIQERILNYRKDGQPFWNQLS ISPVRDETGNVVAFV } \\
\text { GVQTDVTA }\end{array}$ \\
\hline $\begin{array}{l}\text { MTS-LOV48- } \\
58-\mathrm{NZ}\end{array}$ & $\begin{array}{l}\text { MSVLTPLLLRGLTGSARRLPVPRAKIHSLGPPVMASGMIVTDAGAGGSGGALKKELQANKKELAQL } \\
\text { KWELQALKKELAQ }\end{array}$ \\
\hline MTS-LOV48-58 & 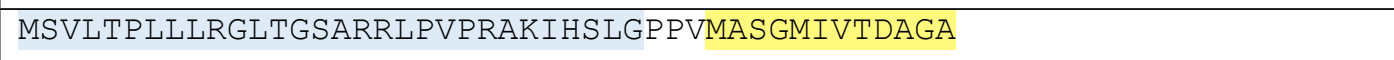 \\
\hline $\begin{array}{l}\text { MTS-CZ- } \\
\text { LOV59-153 }\end{array}$ & $\begin{array}{l}\text { MSVLTPLLLRGLTGSARRLPVPRAKI HSLGPPVGSGMEQLEKKLQALEKKLAQLEWKNQALEKKLA } \\
\text { QGGSGGDQPIVFVNRAFSTITGYAPNEVLGRNARFLQGPQTDAATVARLREAIAAARPIQERI LNY } \\
\text { RKDGQPFWNQLSISPVRDETGNVVAFVGVQTDVTA }\end{array}$ \\
\hline $\begin{array}{l}\text { MTS-LOV48- } \\
\text { 91-NZ }\end{array}$ & $\begin{array}{l}\text { MSVLTPLLLRGLTGSARRLPVPRAKIHSLGPPVMASGMIVTDAGADQP IVFVNRAFSTITGYAPNE } \\
\text { VLGRNARFLQGAGGSGGALKKELQANKKELAQLKWELQALKKELAQ }\end{array}$ \\
\hline MTS-LOV48-91 & $\begin{array}{l}\text { MSVLTPLLLRGLTGSARRLPVPRAKI HSLGPPVMASGMIVTDAGADQP IVFVNRAFSTITGYAPNE } \\
\text { VLGRNARFLQGA }\end{array}$ \\
\hline $\begin{array}{l}\text { MTS-CZ- } \\
\text { LOV92-153 }\end{array}$ & $\begin{array}{l}\text { MSVLTPLLLRGLTGSARRLPVPRAKI HSLGPPVGSGMEQLEKKLQALEKKLAQLEWKNQALEKKLA } \\
\text { QGGSGGQTDAATVARLREAIAAARPIQERI LNYRKDGQPFWNQLSISPVRDETGNVVAFVGVQTDV } \\
\text { TA }\end{array}$ \\
\hline $\begin{array}{l}\text { MTS-LOV48- } \\
137-N Z\end{array}$ & $\begin{array}{l}\text { MSVLTPLLLRGLTGSARRLPVPRAKI HSLGPPVMASGMIVTDAGADQP IVFVNRAFSTITGYAPNE } \\
\text { VLGRNARFLQGPQTDAATVARLREAIAAARP IQERI LNYRKDGQPFWNQLS ISPVRDEGGSGGALK } \\
\text { KELQANKKELAQLKWELQALKKELAQ }\end{array}$ \\
\hline $\begin{array}{l}\text { MTS-LOV48- } \\
137\end{array}$ & $\begin{array}{l}\text { MSVLTPLLLRGLTGSARRLPVPRAKI HSLGPPVMASGMIVTDAGADQP IVFVNRAFSTITGYAPNE } \\
\text { VLGRNARFLQGPQTDAATVARLREAIAAARPIQERI LNYRKDGQPFWNQLS ISPVRDE }\end{array}$ \\
\hline $\begin{array}{l}\text { MTS-CZ- } \\
\text { LOV138-153 }\end{array}$ & $\begin{array}{l}\text { MSVLTPLLLRGLTGSARRLPVPRAKIHSLGPPVGSGMEQLEKKLQALEKKLAQLEWKNQALEKKLA } \\
\text { QGGSGGTGNVVAFVGVQTDVTA }\end{array}$ \\
\hline $\begin{array}{l}\text { SUMO-LOV48- } \\
\text { 91-CaM }\end{array}$ & $\begin{array}{l}\text { MHHHHHHMGSLQDSEVNQEAKPEVKPEVKPETHINLKVSDGSSEIFFK IKKTTPLRRLMEAFAKRQ } \\
\text { GKEMDSLTFLYDGIEIQADQTPEDLDMEDNDI IEAHREQIGGENLYFQGASGMIVTDAGADQPIVF } \\
\text { VNRAFSTITGYAPNEVLGRNARFLQGAGGSGGVSLMADSLTEEQVSEYKEAFSLFDKDGDGQITTK } \\
\text { ELGTVMRSLDQNPSESELQDMINEVDAGSDGT IDFPEFLTMMAREMKYRDTEEEIREVCKVFDRDN } \\
\text { DGFIDAAELRHVMTSI GEKLTDDEVDEMIREADQDGDGRIDYNEFVQLMMQK }\end{array}$ \\
\hline $\begin{array}{l}\text { M13-LOV92- } \\
\text { 153-His }\end{array}$ & $\begin{array}{l}\text { MRSRRLLKKA I DTVRA INKLREGMGGGGSGGQTDAATVARLREA IAAARP IQERI LNYRKDGQPFW } \\
\text { NQLS IS PVRDETGNVVAFVGVQTDVTAHHнHHH }\end{array}$ \\
\hline
\end{tabular}


Table S2. Fluorescence quantum yields with standard deviations from six independent measurements for LOV58insTEV, LOV91insTEV and LOV137insTEV before and after TEV protease cleavage. Fluorescence quantum yield of CagFbFP is $0.36 \pm 0.01$ (Nazarenko et al., 2019).

\begin{tabular}{l|c|c|c} 
Construct & LOV58insTEV & LOV91insTEV & LOV137insTEV \\
\hline Before TEV protease cleavage & $0.36 \pm 0.03$ & $0.35 \pm 0.03$ & $0.36 \pm 0.03$ \\
\hline After TEV protease cleavage & $0.32 \pm 0.04$ & $0.29 \pm 0.03$ & $0.34 \pm 0.03$
\end{tabular}



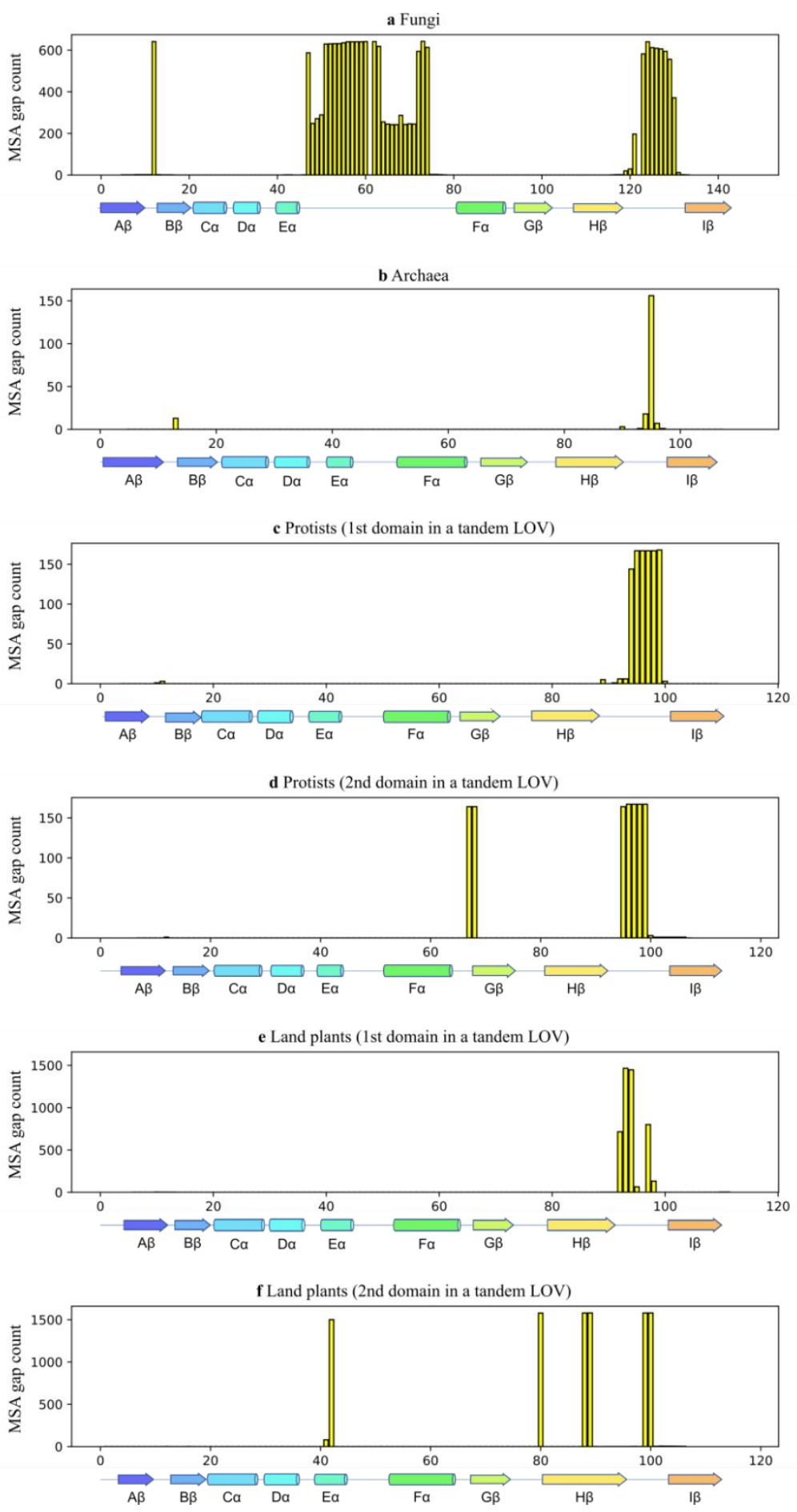

Figure S1. Variation of loop lengths in fungi (a), archaea (b) and tandem LOV domains from protists (c-d) and land plants (e-f), as highlighted by the number of gaps at respective positions in multiple sequence alignments (MSAs). Amino acid positions in respective MSAs and conserved secondary structure elements are shown below the histograms. The sequences were taken from Glantz et al., 2016. Most variation is observed in the $\mathrm{A} \beta-\mathrm{B} \beta, \mathrm{E} \alpha-\mathrm{F} \alpha$, and $\mathrm{H} \beta-\mathrm{I} \beta$ loop lengths. 
a

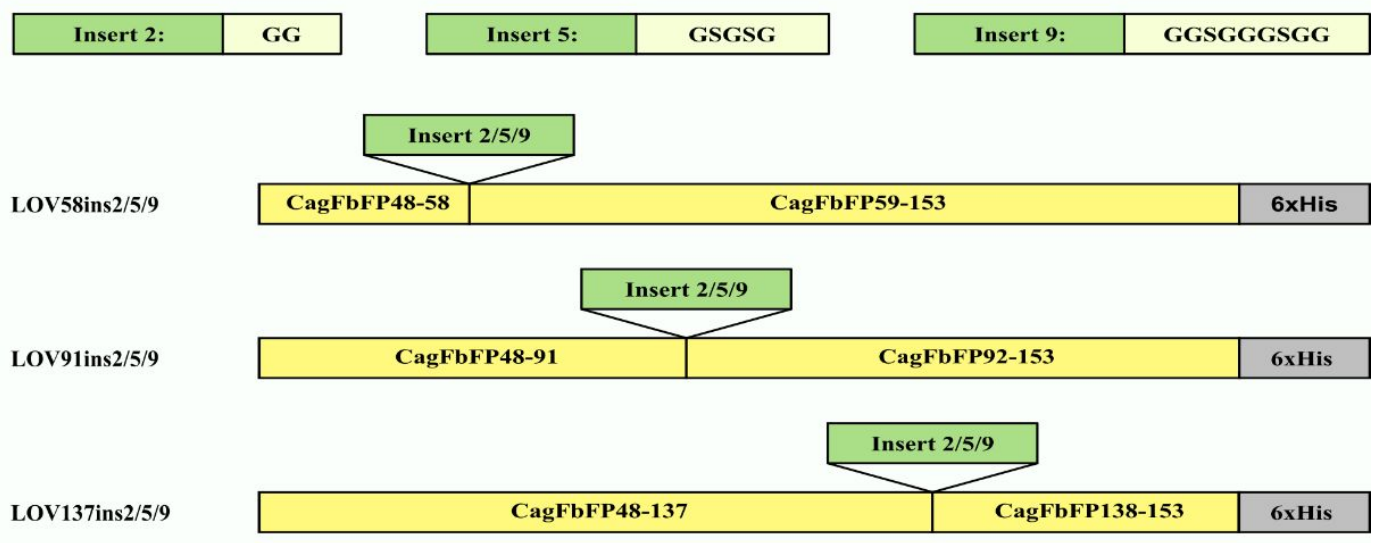

b

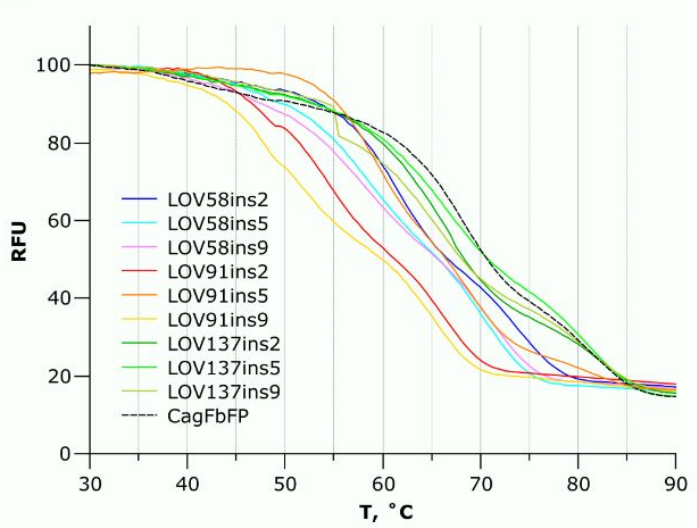

d

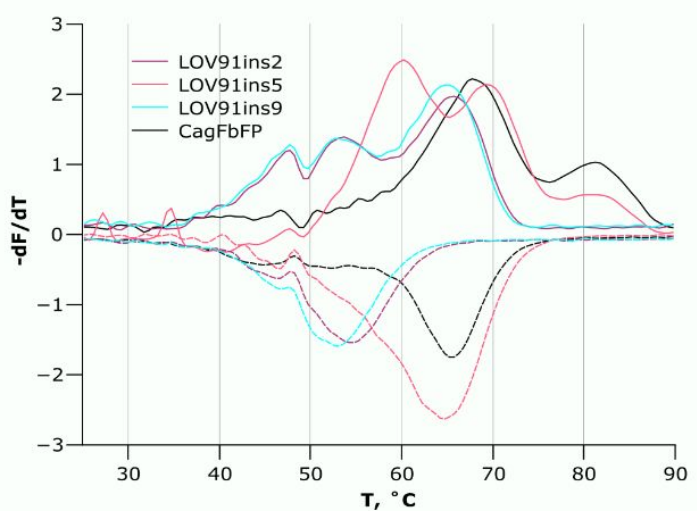

C

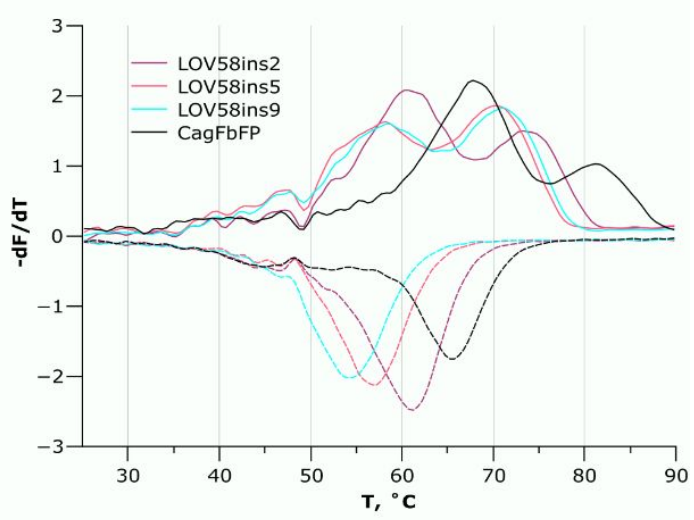

e

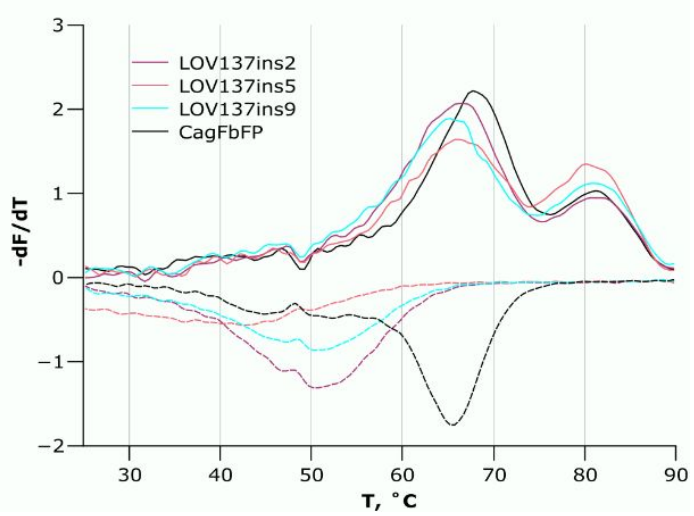

Figure S2. Thermal stability of CagFbFP variants with inserts in different loops. Inserts at all three sites that were tested were tolerated well. (a) Schematic representations of the engineered constructs with flexible inserts. (b) Melting curves of engineered constructs with flexible inserts. Unfolding results in unbinding of flavonoids and quenching of their fluorescence, which is observed as decay in fluorescence intensity. (c,d,e) Derivatives of fluorescence change with temperature for the three different insert sites. The peaks correspond to the transition temperatures. The troughs at $48{ }^{\circ} \mathrm{C}$ are due to the instrument. 

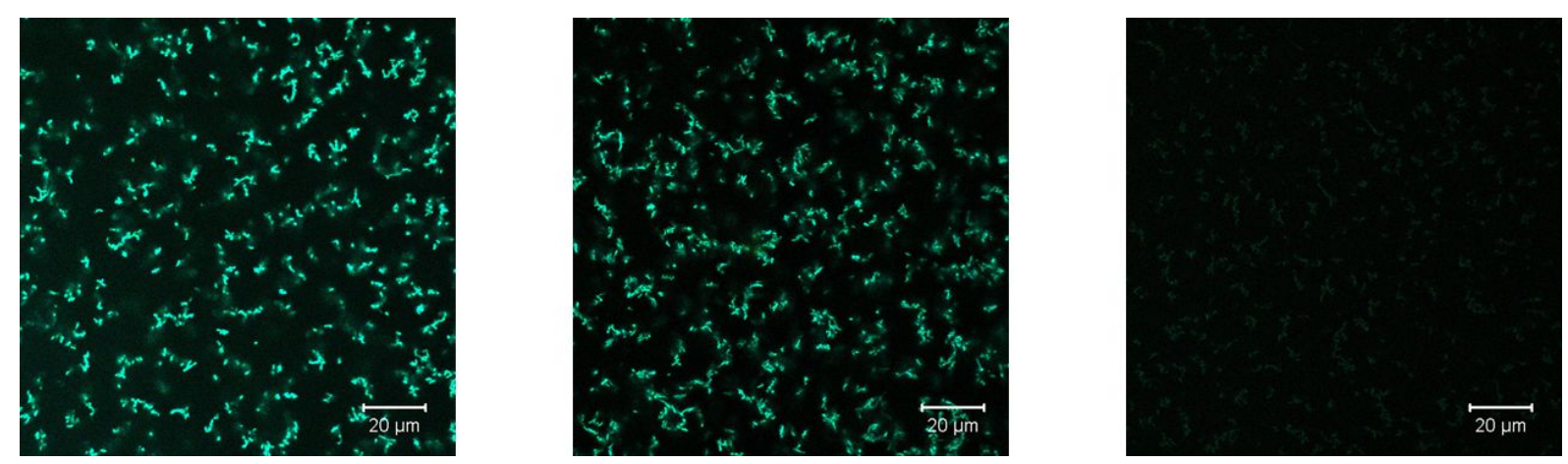

Figure S3. Fluorescence microscopy images of FbFP-transformed E. coli cells. (left) Cells transformed with the plasmid coding the intact protein, CagFbFP. (center) Cells transformed with two plasmids coding interacting fragments, SUMO-LOV48-58-NZ and CZLOV59-153. (right) Cells transformed with two plasmids coding non-interacting constructs, SUMO-LOV48-58 and CZ-LOV59-153, as a negative control. All images are obtained using the same optical settings. Both CagFbFP and split-CagFbFP fluorescence significantly exceeds the autofluorescence of $E$. coli cells. For the constructs with zippers, the observed fluorescence appeared stronger at the cell poles. 